\title{
ATTIVITÀ GLOTTODIDATTICHE WEB-BASED PER STUDENTI ERASMUS
}

\author{
Manuela Cameranesi, Silvia Cañas \& Cecilia Santanché*
}

\begin{abstract}
Presentiamo un'attività glottodidattica per studenti universitari in procinto di partire per il progetto Erasmus in Italia, in Portogallo o in Spagna. La proposta operativa consiste in un WebQuest, ossia una inquiry-oriented activity che prevede il reperimento delle informazioni necessarie per la risoluzione del task assegnato, utilizzando siti web precedentemente selezionati dall'insegnante. L'autenticità dei documenti da consultare consente ai discenti di potenziare la competenza linguistico-comunicativa in campi semantici senz' altro utili per affrontare il periodo di studi all'Estero, attraverso lo svolgimento di un'attività didattica motivante e flessibile, in quanto può essere svolta sia online che in presenza, e adattabile ai diversi livelli di conoscenza della LS.
\end{abstract}

PAROLE CHIAVE: WebQuest; attività web-based; inquiry oriented activity

RESUMO: Apresentamos uma atividade didática aplicada ao ensino das línguas para estudantes universitários prestes a viajar no âmbito do projeto Erasmus para a Itália, Portugal ou Espanha. A proposta operacional consiste em um WebQuest, isto é, uma inquiry-oriented activity que prevê a busca das informações necessárias para a resolução da tarefa dada, utilizando sites da internet anteriormente selecionados pelo professor. A autenticidade dos documentos a serem consultados permite aos discentes potencializar a competência linguístico-comunicativa em campos semânticos sem dúvida úteis para enfrentar o período de estudos no exterior, por meio do desenvolvimento de uma atividade didática motivadora e flexível, pois pode ser realizada tanto a distância quanto em presença, e pode ser adequada aos diferentes níveis de conhecimento da LE.

PALAVRAS CHAVE: WebQuest; atividades web-based; inquiry oriented activity

ABSTRACT: We present a didactic activity for university students who are about to leave for the Erasmus project in Italy, Portugal or Spain. The operative proposal consists in a WebQuest, that is an inquiry-oriented activity that provides the retrieval

* Università "G. d'Annunzio", Chieti-Pescara (Italia)

manuelacameranesi@gmail.com, silvia.canas@unich.it, cecisantanche71@gmail.com DOI: http://dx.doi.org/10.11606/issn.2238-8281.v0i38p5-16 
of the information necessary for the resolution of the assigned task, using websites previously selected by the teacher. The authenticity of the documents to be consulted allows learners to enhance their linguistic and communicative competence in semantic fields which are certainly useful for addressing the period of study abroad, through the performance of a motivating and flexible teaching activity, as it can be carried out both online and in attendance, and is adaptable to different levels of LS knowledge.

KEY WORDS: WebQuest; didactic activities; web-based; inquiry oriented activity 


\section{Il WebQuest nei corsi di lingua per gli outgoing students ${ }^{1}$}

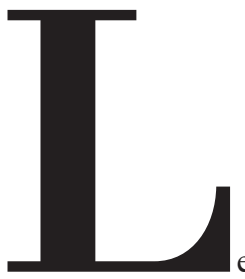

e recenti evoluzioni nell'ambito delle Tecnologie dell'Informazione e della

Comunicazione (TIC) hanno apportato un cambiamento significativo nel reperimento e nella diffusione delle informazioni, facilitandone l'accesso. Di conseguenza, anche nell' ambito della didattica delle lingue si è avvertito il bisogno di integrare le tradizionali attività didattiche con un supporto informatico, determinando un ripensamento degli approcci educativi e generando un ambiente di apprendimento interattivo motivante e adeguato alle esigenze degli studenti. La conferma di questa impellente necessità di adattarsi ai cambiamenti apportati dal settore della tecnologia arriva anche dalla pubblicazione di documenti ufficiali divulgati da organismi internazionali quali l'OCSE, l'UNESCO e la Commissione Europea, ove viene incentivato nei sistemi educativi il ricorso alla "più avanzata tecnologia informatica", ma allo stesso tempo si raccomanda un'adeguata alfabetizzazione digitale (o digital literacy) nella progettazione curricolare, ossia un uso intelligente di questo "strumento cognitivo" che consente lo sviluppo dell'autonomia e di nuove forme di ragionamento in prospettiva di un "lifelong learning" (CONSIGLIO D'EUROPA, 2002, p. 3). Il termine digital literacy fu coniato da Paul Glister, per indicare le competenze cognitive e culturali per un utilizzo appropriato e consapevole della Rete (D'ANGELO, 2012), allo scopo di sviluppare anche competenze trasversali connesse a quella digitale (ad esempio, la conoscenza delle lingue e della matematica o la consapevolezza culturale), al fine di assicurare inclusione e partecipazione attiva nella società moderna (CONSIGLIO D'EUROPA, 2002).

I nativi digitali, nati e cresciuti con un background dominato dalla tecnologia, tendono ad affidarsi passivamente agli strumenti tecnologici, dimostrando spesso uno scarso senso critico nell'uso delle risorse del Web (CILIBERTI, 2012). Questo comportamento è dovuto anche alla struttura non lineare della rete, che può causare disorientamento nella ricerca e nel reperimento delle informazioni più appropriate. Inoltre, non è da sottovalutare il fatto che i contenuti presenti in Internet possono essere pubblicati da svariate tipologie di utenti, anche da chi non è professionista in un dato settore, e potrebbe dunque diffondere informazioni false (MARCH, 1998).

$1 \quad$ Il presente contributo è stato concepito ed elaborato in collaborazione tra le autrici; tuttavia, per la stesura del lavoro, i §§1 e 2 sono da attribuire a Manuela Cameranesi, il § 3 a Silvia Cañas e il § 4 a Cecilia Santanchè. 
Uno strumento che può servire a potenziare la digital literacy è il WebQuest. Si tratta di un'attività web-based ideata dal pedagogista statunitense Bernie Dodge (1995) e definita come "an inquiry-oriented activity in which some or all of the information that learners interact with comes from resources on the Internet"'. L'uso di questo strumento è finalizzato all'apprendimento linguistico e al potenziamento di abilità cognitive e digitali, attraverso il ricorso a materiali autentici in lingua straniera reperibili nel World Wide Web. Pertanto, ciò che distingue questa modalità di apprendimento basata sull'indagine da quelle tradizionali è l'esplicita richiesta di disporre di Internet per portare a termine il compito assegnato dall'insegnante, nell'intento di integrare le potenzialità del Web con le attività didattiche implementate per il raggiungimento di determinati learning objectives.

\section{Il WebQuest come attività pre-Erasmus}

In questo contributo proponiamo esempi concreti di WebQuest pensati per studenti Erasmus Outgoing verso l'Italia, la Spagna e il Portogallo, tuttavia si precisa che tali tipologie di attività possono essere applicate anche in altri contesti di mobilità e progetti internazionali.

I siti che gli apprendenti dovranno visitare e i documenti che dovranno leggere riguardano ambienti e situazioni reali che i discenti si troveranno a vivere e a fronteggiare durante il loro soggiorno all'Estero, pertanto si può prevedere che la motivazione sarà molto alta. Inoltre, l'autenticità delle pagine web ha un risvolto positivo anche per la competenza linguistico-comunicativa dei discenti, i quali hanno l'opportunità di incontrare ed interiorizzare locuzioni e unità lessicali di campi semantici di sicura utilità.

Per garantire ordine nell'esecuzione del lavoro, il docente divide la classe in gruppi, suddividendo e personalizzando i compiti al fine di accertarsi che ogni discente apporti il proprio contributo. Questo processo mira ad incentivare l'apprendimento cooperativo attraverso la strategia del problem solving, usufruendo delle risorse presenti in Rete e collaborando per risolvere un compito finale condiviso (NARDON-SCHMID, 2011).

In altri termini, la didattica per task implica un' attività in cui la lingua straniera è impiegata per raggiungere obiettivi extralinguistici reali di vario genere. Nel progetto che presentiamo si chiede agli apprendenti di portare a termine compiti effettivi che potranno applicare concretamente nella loro esperienza all'estero, come selezionare un alloggio adeguato o individuare i mezzi di trasporto più convenienti per raggiungere un determinato luogo. In questo modo, i discenti vengono messi nella condizione di dover reperire, comprendere e rielaborare informazioni in base al task assegnato.

In linea con gli approcci comunicativi e umanistico-affettivi, la didattica basata sul task procede quindi da una visione learner-centred nella quale si prendono in considerazione $\mathrm{i}$ diversi stili di apprendimento dei discenti (ELLIS, 2003) che sono liberi di affrontare il compito nella maniera più vicina al proprio modo di operare, che può essere riflessivo o intuitivo (analitico/globale), seguire un'idea o basarsi su fatti (ideativo/esecutivo), tollerare o no le ambiguità,

2 March (2003/2004), collaboratore di Dodge, propone anche un'altra definizione di WebQuest: "A WebQuest is a scaffolded learning structure that uses links to essential resources on the World Wide Web and an authentic task to motivate students' investigation of a central, open-ended question, development of individual expertise and participation in a final group process that attempts to transform newly acquired information into a more sophisticated understanding." 
essere legato o no al contesto e autonomo nei processi di studio (BALBONI, 2015). Infatti, l'orientamento all'azione e la personalizzazione del compito, progettato proprio per assolvere una funzione utile agli studenti, comportano la partecipazione attiva degli apprendenti, stimolano la ricerca autonoma delle soluzioni più opportune per eseguire il compito stabilito.

La nostra attività è pensata seguendo una modalità blended, ossia prevedendo alcune lezioni in presenza, ma anche ore di lavoro autonomo, svolte a distanza. Allo scopo di evitare la selezione di informazioni fuorvianti e superflue, vengono riportate domande mirate e link precedentemente verificati dall' insegnante (v. § 3). In questo senso, il docente, chiedendo di eseguire un task specifico nella lingua target, avrà la possibilità di valutare la crescita della competenza linguistica degli allievi e la loro capacità di reperire criticamente e rielaborare efficacemente dati nel Web. Il docente assume il ruolo di facilitatore, fornendo le indicazioni da seguire, grazie anche all'ausilio della struttura stessa del WebQuest, strumento didattico flessibile relativamente alla durata e alla modalità di svolgimento del lavoro (ossia in presenza, a distanza o in modalità blended), ma che contiene una struttura fissa utile come scaffolding ${ }^{3}$ e una guida per i discenti.

La creazione di un WebQuest risulta, infatti, assai intuitiva ed è possibile usufruire anche di authoring software gratuiti per realizzarla. Nel progetto in questione abbiamo utilizzato il portale Questgarden ${ }^{4}$, che non necessita di conoscenze tecnico-informatiche specifiche. L'uso di questo sito permette agli insegnanti di elaborare agevolmente uno o più WebQuest, pubblicarli e inviare il link di riferimento ai propri studenti; cliccando gli apprendenti accedono ad una pagina contenente parole attive sulla parte sinistra dello schermo (nell'esempio riportato nella figura 1 esse sono: introduzione, compito, procedimento, valutazione, conclusione, ringraziamenti e pagina insegnanti) e 'sfogliando' le pagine del WebQuest, passo dopo passo, svolgono il compito stabilito dal docente.

\section{Esempio di WebQuest per gli Outgoing students verso la Spagna}

Tra gli obiettivi del WebQuest risulta di fondamentale importanza suscitare motivazione, al fine di incentivare gli studenti a portare a termine il compito assegnato. La prima sezione che gli studenti vedranno, l' "introduzione", è orientata proprio in questa direzione: in essa si propongono infatti frasi motivanti e foto delle città scelte come mete del progetto di mobilità discente, così da destare curiosità.

Il compito prevede la duplice creazione di una guida realizzata con PowerPoint (max. 10 diapositive) e di un video (max. 3 minuti), registrato utilizzando l'applicazione Flipgrid: il

3 Si ritiene opportuno precisare che sia Dodge che March reputano che il WebQuest possa essere un punto di partenza per stimolare un certo tipo di ragionamento critico, così che questo processo venga interiorizzato e diventi un atteggiamento e un approccio ai problemi non solo in un'ottica interdisciplinare nel periodo di formazione, ma anche per il futuro (MARCH, 2003/2004).

4 Questgarden offre la possibilità di usufruire della versione di prova gratuita per trenta giorni, consentendo di realizzare il proprio WebQuest e scaricarlo, dopo averlo concluso, come file Zip. È quindi possibile creare il proprio account iscrivendosi alla versione trial, destinata all'uso di una sola persona che abbia compiuto diciotto anni. Alla scadenza dei trenta giorni non sarà più possibile modificare il proprio WebQuest che potrà tuttavia essere scaricato e rimarrà pubblicato e disponibile online solo per un anno. Nella versione a pagamento, una volta creato, il WebQuest può essere ripristinato in qualsiasi momento. Si segnalano anche altri siti ove sono presenti ulteriori esempi e informazioni relativi al WebQuest: http://webquest.org/index.php; http://zunal.com/; http://www.bibliolab.it/webquest.htm. 
primo prodotto dovrà essere presentato in classe in lingua straniera, in base al livello linguistico degli apprendenti e alle nozioni acquisite, allo scopo di ampliare le conoscenze collettive ma anche di avviare un dibattito, mentre il video verrà inviato al docente, così da permettere una valutazione individuale della competenza linguistica e della capacità di saper argomentare le proprie scelte. Gli studenti avranno due settimane per elaborare il PowerPoint e una settimana per registrare il video.

Nella sezione del "procedimento" vengono sviscerati i punti da seguire per la realizzazione dei due compiti. Gli studenti sono divisi in quattro gruppi in base alle città scelte e, all'interno di ciascun gruppo, ogni alunno ha un proprio argomento da sviluppare, estrapolando informazioni dai link assegnati dall'insegnante sui seguenti temi: mezzi di trasporto, alloggi, corsi universitari e svago. Data l'ampiezza dei contenuti presenti nei link proposti, vengono poste delle domande mirate, così da guidare gli studenti e fornire loro una sorta di traccia nella lettura dei vari siti. I quesiti risultano essere molto specifici proprio per verificare l'abilità degli apprendenti di "muoversi" all'interno della rete, selezionando in maniera critica solo i dati richiesti dal task. Viene per esempio chiesto loro di:

- tracciare percorsi per spostarsi da uno specifico posto all'altro all'interno della città (ad esempio dall'aeroporto alla stazione);

- selezionare il mezzo di trasporto più conveniente per muoversi;

- trovare determinate linee degli autobus e i relativi costi dei biglietti;

- decidere quale tipo di alloggio risulti più conveniente;

- definire i requisiti per poter usufruire del servizio di residenza universitaria offerto dall'Azienda Regionale per il Diritto allo Studio Universitario;

- trovare l'appartamento più vicino all'università e riportare il tragitto suggerito da Google Maps e le immagini della casa;

- elencare i corsi di laurea offerti da uno specifico dipartimento;

- trovare i corsi di laurea che nella loro attività formativa prevedono l'insegnamento della letteratura italiana e riportare in quale semestre si svolgono le lezioni;

- esporre i contenuti di un corso specifico (ad esempio, di Letteratura Italiana) e il programma, spiegando come sono state reperite le informazioni attraverso una mail (max. 50 parole), inserendo anche il link e lo screenshot del programma;

- elencare le strutture convenzionate con l'università;

- cercare concerti e mostre previsti per l'estate e i relativi costi dei biglietti.

Anche relativamente alla registrazione del video è fornita una traccia da seguire:

- Perché hai deciso di fare l'Erasmus in questo Paese?

- Perché hai scelto questa città per il tuo Erasmus?

- Pensi che siano offerti dei servizi vantaggiosi agli studenti? 
- Pensi che sia semplice vivere e studiare nella città che hai scelto? Perché?

La "valutazione" dei prodotti finali è individuale e di gruppo per un totale di massimo 10 punti: la prima valuta il ruolo che ciascuno dei membri del gruppo ha svolto, mentre la seconda prende in considerazione la forma in cui è stato eseguito il lavoro previsto del task. Allo scopo di garantire trasparenza nel giudizio, i discenti possono visualizzare tutti i criteri per cui verranno valutati.

Nella conclusione è ribadito il risultato del WebQuest, si esprime un giudizio globale sul lavoro svolto, enfatizzando la consapevolezza e i risultati raggiunti, e vengono inoltre forniti ulteriori link di approfondimento.

Figura 1: Esempio di conclusione nel WebQuest sull'Erasmus in Spagna

\section{Un semestre Erasmus en España}

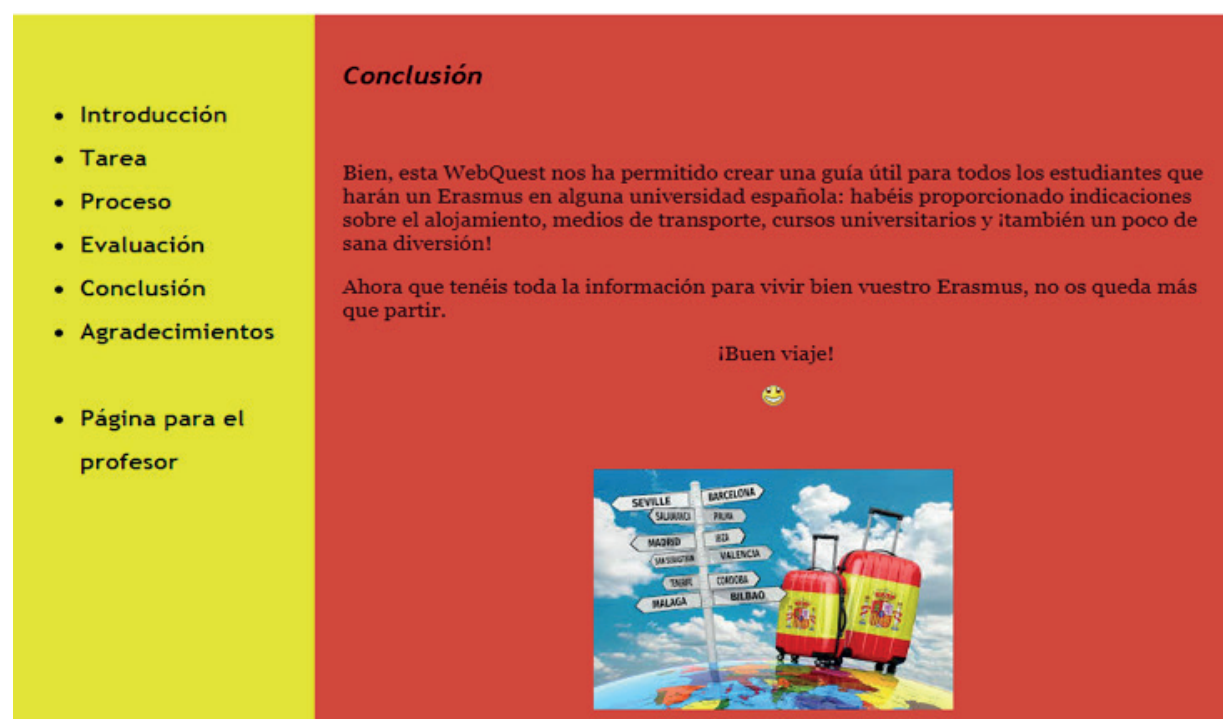

In aggiunta alla pagina rivolta agli studenti è presente anche una parte per gli insegnanti, ove vengono dati dettagli glottodidattici sul procedimento da seguire per svolgere l'attività in questione. Si specificano il target per cui abbiamo pensato il WebQuest e la visione glottodidattica di tale proposta operativa, ma, allo stesso tempo, si precisa che l'attività che proponiamo funge da esempio, quindi i dati inseriti possono essere modificati a piacimento e in base alle proprie esigenze. 
Di seguito si riporta l' "introduzione della pagina dell'insegnante", realizzata nel WebQuest "Erasmus in Italia":

Figura 2: Esempio di “introduzione alla pagina insegnanti” nel WebQuest sull'Erasmus in Italia

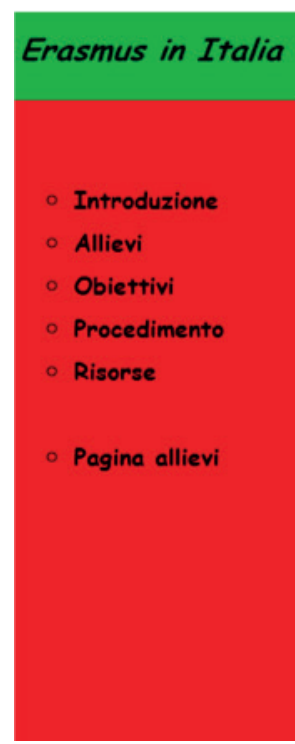

by Manuela Cameranesi

Introduzione insegnanti

Questo WebQuest è pensato per studenti Erasmus Portoghesi e Spagnoli Incoming verso l'Italia e studenti Erasmus Italiani Outgoing verso il Portogallo e la Spagna.

Attraverso la strategia del problem solving e proponendo un metodo di apprendimento cooperativo, gli studenti si troveranno ad analizzare materiali autentici allo scopo di reperire informazioni utili per il loro soggiorno nella città scelta come meta Erasmus.

L'insegnante avrà la possibilità di verificare la conoscenza linguistica degli apprendenti (grammatica, lessico e fluency), valutare la capacità di produzione (scritta e orale) e potenziare la la digital reading e la digital literacy, ossia l'abilità di selezionare criticamente $i$ dati presenti in Rete.

Nb. Il presente WebQuest viene presentato come esempio di attività web-based, ma si desidera precisare che $\mathrm{i}$ dati inseriti possono variare in base alle esigenze specifiche dell'insegnante e della classe.

Ancora, nella Pagina dell'insegnante sono descritti in modo approfondito gli obiettivi che il WebQuest si prefigge e su cui si focalizzerà la valutazione del docente: 
Figura 3: Esempio di “obiettivi” riportati nella pagina insegnanti nel WebQuest sull'Erasmus in Italia

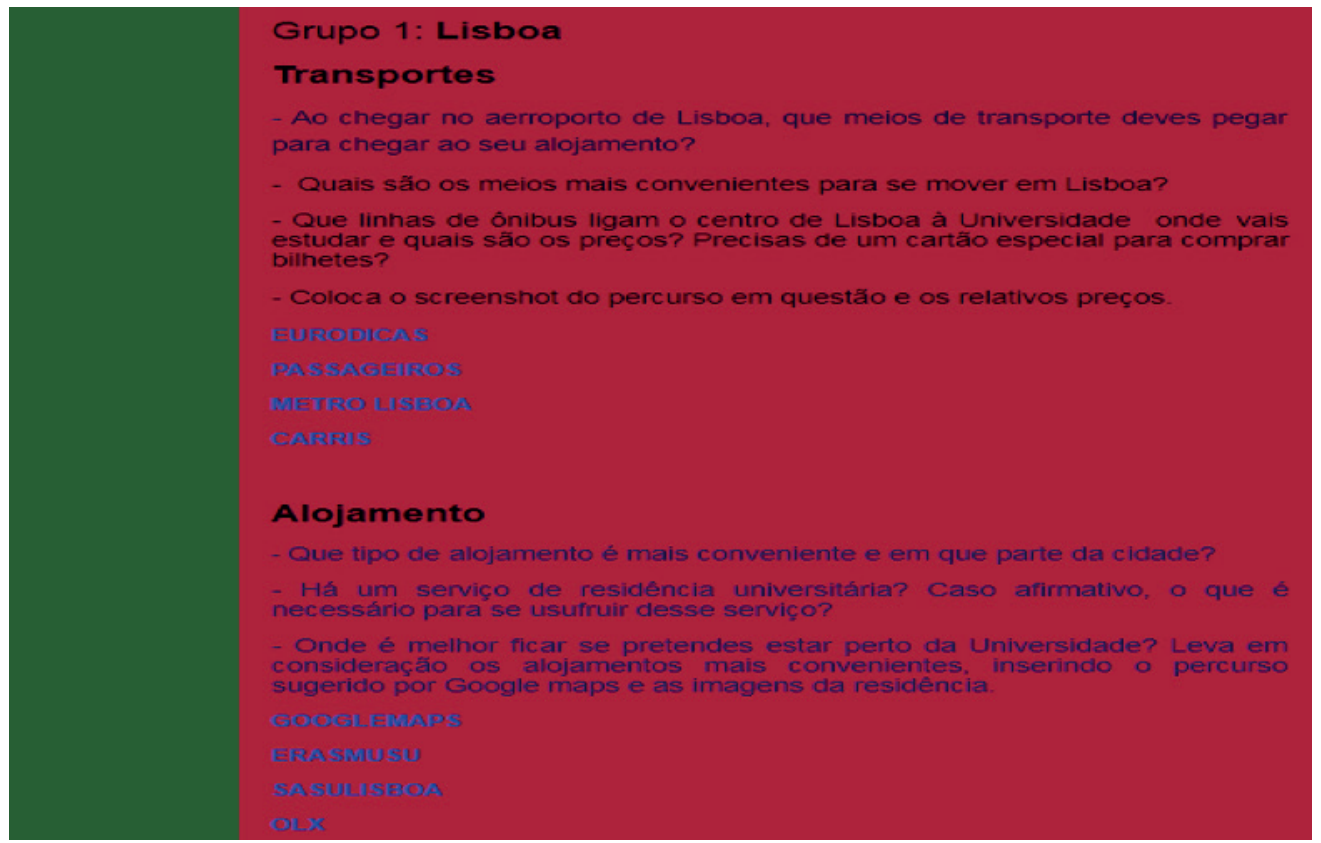

\section{Esempio di WebQuest per gli Outgoing Students verso il Portogallo}

In questa sezione presentiamo un esempio di WebQuest per studenti Erasmus Italiani Outgoing verso il Portogallo, sviluppato come negli altri casi, al fine di migliorare l'inserimento nel Paese.

Il contesto d'uso per cui è pensato tale progetto è il corso di lingua portoghese che le università italiane offrono prima della partenza, nel quale viene insegnata la lingua e vengono fornite informazioni culturali. Il WebQuest risulta essere un primo vero contatto con il portoghese attraverso l'analisi di siti autentici. Ipotizziamo che il presente lavoro possa essere introdotto alla classe durante le prime lezioni, anche per evidenziare le differenze tra il portoghese del Portogallo e quello del Brasile.

Nell' "introduzione" abbiamo utilizzato il pronome inclusivo sottinteso (Vamos conhe- 
cer Portugal com o Projeto Erasmus!!!) con l'obiettivo di rivolgerci agli studenti in modo più diretto e facendoli sentire parte di un gruppo, che andranno poi a formare per risolvere il compito assegnato, ossia la presentazione PowerPoint e il video con Flipgrid.

Nel processo abbiamo suddiviso i compiti e stabilito i tempi per ogni parte dell'attività. Conseguentemente, abbiamo domandato di risolvere quesiti che si porranno effettivamente per il programma di scambio. Per garantire la consultazione di pagine web attendibili abbiamo fornito loro link reali e autentici:

Figura 4: Esempio di procedimento nel WebQuest sull'Erasmus in Portogallo

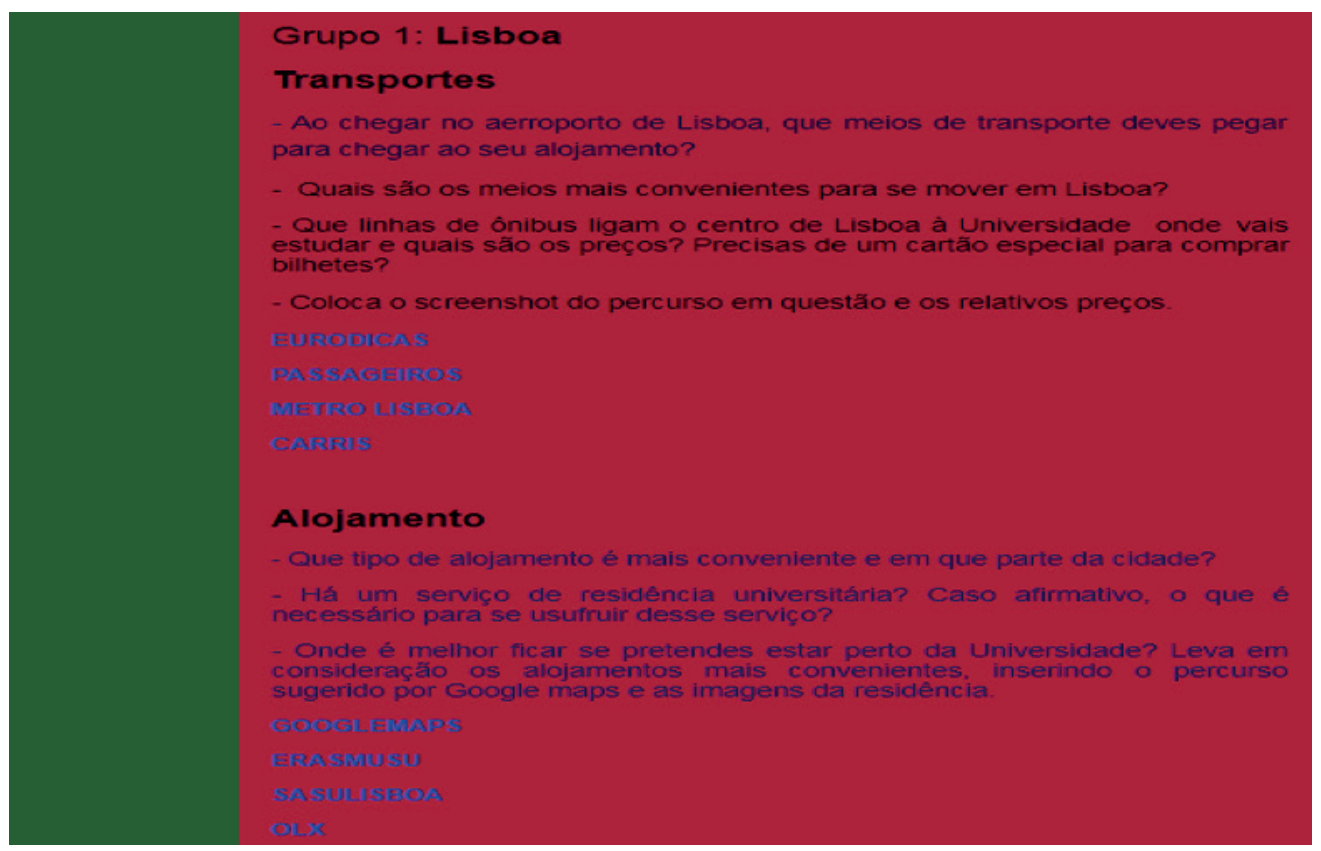

Come si nota, per i trasporti sono stati inseriti i link dei mezzi locali che effettivamente sono presenti nelle città prescelte, mentre per ciò che concerne l'Università e le attività di svago abbiamo tenuto in considerazione i precedenti soggiorni Erasmus.

In conclusione, avendo avuto esperienza di corsi di preparazione agli scambi internazionali, riteniamo che un'attività come il WebQuest possa risultare molto utile per lo sviluppo linguistico e cognitivo dei discenti, tuttavia siamo consapevoli della scarsità di tempo a disposizione durante tale corso. Pertanto, riteniamo che sarebbe opportuno dedicare un'ora a settimana allo svolgimento in classe del WebQuest, così da controllare il lavoro condotto dagli studenti e 
offrire delucidazioni laddove ce ne fosse bisogno.

Inoltre si potrebbe migliorare l'effetto sortito dal WebQuest se questo fosse abbinato alle normali attività svolte in corsi simili, come ad esempio: (a) ampliamento del lessico, scegliendo parole pertinenti alle situazioni proposte nel WebQuest (ovvero relative a mezzi di trasporto, alloggio, corsi universitari e svago) e memorizzando le espressioni tipiche della lingua target (come ad esempio le espressioni idiomatiche) che si incontreranno nelle pagine web; (b) sviluppo delle abilità attraverso situazioni reali: ascolto, dialogo, roleplay direzionato, lettura di testo da manuali, attività di problem solving. Tali attività potrebbero essere svolte successivamente allo studio dei link suggeriti nel WebQuest, ossia dopo aver familiarizzato e interiorizzato termini utili.

Relativamente al video Flipgrid ${ }^{5}$ in cui vengono esposte le informazioni raccolte e si giustificano le proprie scelte, un ulteriore fattore di motivazione potrebbe essere quello di inviare lo stesso a coetanei madrelingua, così da avere un riscontro sulla propria conoscenza linguistica e sulla selezione di dati davvero utili e adeguati.

\section{Conclusioni}

L'avvento delle TIC nella didattica delle lingue ha condotto ad un rinnovamento degli ambienti di apprendimento, affiancando alle tradizionali attività didattiche nuove metodologie e strumenti.

Le attività didattiche web-based, come i WebQuest proposti qui, stimolano la partecipazione degli studenti e consentono un rapido accesso al materiale offerto dalla rete. Oltre a ciò, questa proposta task-oriented è volta ad incentivare l'apprendimento cooperativo, giacché si chiede agli apprendenti di risolvere un compito comune, attraverso la consultazione del World Wide Web. Il ricorso a siti autentici e la richiesta che il docente fa ai propri studenti di reperire informazioni specifiche permette di sviluppare la digital literacy, rendendo i discenti più autonomi nel giudizio dei dati e dell'attendibilità dei siti web. Tra gli effetti positivi apportati dalla visita delle pagine web si sottolinea l'ampliamento della conoscenza linguistico-comunicativa anche a campi semantici relativi agli ambienti e alle situazioni nelle quali gli studenti si troveranno durante il loro percorso.

Oltre al potenziamento della competenza linguistica, vengono incrementate capacità cognitive superiori come lo sviluppo del pensiero critico nella ricerca di informazioni pertinenti, l'abilità di pianificare strategie di problem solving e la capacità di prendere decisioni appropriate al compito (MARCH, 1998).

Infine, la scelta di tale strumento è dettata anche dalla sua flessibilità, poiché gli insegnanti hanno la possibilità di applicarlo in modalità blended, quindi online e in presenza, e vi è l'opportunità di personalizzare i compiti nel rispetto dei diversi livelli di competenza e stili di apprendimento degli studenti.

5 L'Università “G. d'Annunzio" ha già sperimentato Flipgrid con l'Università di Porto, con la quale è stato stipulato un accordo bilaterale. L'obiettivo è quello di facilitare l'accoglienza degli studenti Erasmus Incoming (sia in Portogallo che in Italia), attraverso una prima presentazione a distanza in lingua straniera e una descrizione dei luoghi più importanti. 


\section{Riferimenti bibliografici}

BALBONI, P. Le sfide di Babele. Insegnare le lingue nelle società complesse. Torino: UTET, 2015. CARLONI, G. Insegnare Italiano a stranieri. Percorsi operativi. Milano: Franco Angeli., 2015.

CILIBERTI, A. Glottodidattica. Per una cultura dell'insegnamento linguistico. Roma: Carocci Editore, 2012.

CONSIGLIO D'EUROPA. Quadro comune europeo di riferimento per le lingue: apprendimento insegnamento valutazione. Firenze: La Nuova Italia, 2002.

D'ANGELO, M. Nuove tecnologie per la didattica delle lingue e della traduzione. Roma: Aracne, 2012.

D'ANGELO, M. Considerazioni glottodidattiche sul concetto di digital literacy. In. AGRESTI, G. \& PALLINI, S. Migrazioni tra disagio linguistico e patrimoni culturali. Roma: Aracne, 2015, p. 115.

DODGE, B. "Some thought about WebQuests". http://webquest.org/sdsu/about_webquests.html Accesso: 22/07/2018.

ELLIS, R. Task-based language Learning and Teaching. Oxford: Oxford University Press, 2003.

MARCH, T. "Why WebQuests". http://tommarch.com/writings/why-webquests/ Ultimo accesso: 23/07/2018.

MARCH, T. "What WebQuests are (really)". http://tommarch.com/writings/what-webquests-are/

NARDON-SCHMID, E. Didattica del lessico e nuove tecnologie. In: DI SABATO, B.\& MAZZOTTA, P. (orgs.), Linguistica e didattica delle lingue e dell'inglese contemporaneo. Studi in onore di Gianfranco Porcelli. Lecce: Pensa Multimedia, 2011, p. 413.

Recebido em: 11/09/2019

Aprovado em: 22/11/2019 